\title{
Editorial to the Special Issue on the Principles of Advanced Discrete Simulation (PADS)
}

Welcome to this special issue of ACM TOMACS on the Principles of Advanced Discrete Simulation (PADS), which is based on selected articles from the 2018 ACM SIGSIM-PADS Conference. This is the flagship conference of the ACM's Special Interest Group on Simulation and Modeling (SIGSIM), whose 2018 edition was held at the University of Rome La Sapienza, on May 23-25, 2018. This special issue is edited by Georgios Theodoropoulos, Alessandro Pellegrini, and Francesco Quaglia, who served as the conference program and general chairs, and were responsible for the proceedings of the conference.

The articles appearing in this special issue have been selected also thanks to the help of Prof. Adelinde Uhrmacher, who acted as the Editor in Chief of ACM TOMACS until August 2019. The outcome of the selection process identified seven articles, top ranked in the review process of PADS 2018, as possible candidates for this special issue. The authors of three of them have been able to meet the deadlines for submitting the substantially extended versions of the original conference articles, while the others were not able to produce sufficiently new results on time to meet the special issue schedule. The submission process for this special issue started in November 2018, and the final decisions for all the involved articles have been made by November 2019, with a very minimal delay with respect to the originally planned schedule. The articles submitted to this special issue and having the organizers of PADS 2018 as authors have been handled directly by Prof. Adelinde Uhrmacher. As an additional mean for keeping the quality of the articles accepted in this special issue at excellent levels, the invited authors had to provide an extension plan of their original conference articles, the adequacy of which has been early assessed by the Guest Editors and by the TOMACS Editor in Chief. Also, a minimum of three reviewers peer reviewed the submitted articles.

The special issue finally consists of three articles. The first article [Rahman and Kemper 2020] focuses on Markov models, which are commonly used in the context of modeling and simulation of dynamic systems. The objective of the article is to investigate the role of specific properties of a discrete-time Markov chain for the purpose of time-series analysis. The properties of interest include entropy and the second-largest eigenvalue. One relevant achievement by the authors is that these properties are affected in a non-independent manner by the presence of trends or periodicity. The article has a strong theoretical focus, but it also shows the potential for pragmatical applications, for example in the study of processes' similarities.

The second article [Andelfinger et al. 2020] addresses the issue of making microscopic traffic simulation more efficient. The idea is to avoid time-stepped state updates of the involved entities. Rather, an approach is provided based on the identification of independent updates among entities. The introduction of a fast-forward state update function is the mean exploited to support

\footnotetext{
ACM Reference format: 8 (March 2020), 2 pages.

https://doi.org/10.1145/3381903

(C) 2020 Copyright held by the owner/author(s).

1049-3301/2020/03-ART8

https://doi.org/10.1145/3381903
}

Francesco Quaglia, Georgios Theodoropoulos, and Alessandro Pellegrini. 2020. Editorial to the Special Issue on the Principles of Advanced Discrete Simulation (PADS). ACM Trans. Model. Comput. Simul. 30, 2, Article 
the accelerated simulation execution. Noteworthy, the proposed solution allows speeding up the simulation model execution without reducing the level of details in the model.

Finally, the third article [Principe et al. 2020] addresses the issue of bridging shared-memory programming and distributed execution of parallel discrete event simulation models. The article introduces a middleware-based architecture which implements a distributed system capable of supporting speculative distributed executions of simulation models originally conceived and coded for a shared-memory environment. The advantage is the avoidance of explicit message-based interactions across concurrent simulation objects-at the level of the application code-while still seamlessly enabling the exploitation of large-scale distributed-memory platforms, such as clusters of virtual machines in the Cloud. Mechanisms to dynamically tune middleware-level parameters, on the basis of the specific simulation workload, are also provided. The effectiveness of this proposal is demonstrated via experiments carried out on top of both private and public Cloud infrastructures.

\section{REFERENCES}

Philip Andelfinger, Yadong Xu, David Eckhoff, Wentong Cai, and Alois Knoll. 2020. Fidelity and performance of state fast-forwarding in microscopic traffic simulations. ACM Transactions on Modeling and Computer Simulation (2020).

Matteo Principe, Tommaso Tocci, Pierangelo Di Sanzo, Francesco Quaglia, and Alessandro Pellegrini. 2020. A distributed shared-memory middleware for speculative parallel discrete event simulation. ACM Transactions on Modeling and Computer Simulation (2020).

Atiqur Rahman and Peter Kemper. 2020. Simulation study to identify the characteristics of Markov chain properties. ACM Transactions on Modeling and Computer Simulation (2020).

Francesco Quaglia

Editor-in-Chief

Georgios Theodoropoulos Alessandro Pellegrini

Guest Editors 